\title{
Persistent expression of Twist1 in chondrocytes causes growth plate abnormalities and dwarfism in mice
}

\author{
ROSA M. GUZZO', VIKTORIA ANDREEVA ${ }^{2}$, DOUGLAS B. SPICER ${ }^{*, 2}$ and M. HICHAM DRISSI*,1 \\ ${ }^{1}$ Department of Orthopaedic Surgery, University of Connecticut Health Center, Farmington, CT and \\ ${ }^{2}$ Center for Molecular Medicine, Maine Medical Center Research Institute, Scarborough, ME, USA
}

\begin{abstract}
Evidence from various in vitro gain and loss of function studies indicate that the bHLH transcription factor Twist 1 negatively regulates chondrocyte differentiation; however limited information regarding Twist 1 function in postnatal cartilage development and maintenance is available. Twist 1 expression within the postnatal growth plate is restricted to immature, proliferating chondrocytes, and is significantly decreased or absent in hypertrophic chondrocytes. In order to examine the effect of maintaining the expression of Twist 1 at later stages of chondocyte differentiation, we used type II collagen Cre (Col2-Cre) mice to activate a Cre-inducible Twist1 transgene specifically in chondrocytes (Col2-Twist1). At two weeks, postnatal growth was inhibited in Col2-Twist1 mice, as evidenced by limb shortening. Histological examination revealed abnormal growth plate structure, characterized by poor columnar organization of proliferating cartilaginous cells, decreased cellularity, and expansion of the hypertrophic zone. Moreover, structural defects within the growth plates of Col2-Twist 1 transgenic mice included abnormal vascular invasion and focal regions of bony formation. Quantitative analysis of endochondral bone formation via micro-computed topography revealed impaired trabecular bone formation in the hindlimbs of Col2-Twist1 transgenic mice at various timepoints of postnatal development. Taken together, these findings indicate that regulated Twist 1 expression contributes to growth plate organization and endochondral ossification to modulate postnatal longitudinal bone growth.
\end{abstract}

KEY WORDS: twist1, chondrocyte, growth plate

Transition of chondrocytes from proliferation to terminal maturation within the growth plate is vital for longitudinal bone growth. Proliferating, immature chondrocytes synthesize an extracellular matrix (ECM) abundant in type II collagen and aggrecan. As cells differentiate, they mature into hypertrophic, postmitotic chondrocytes that enrich the ECM in type X collagen. Following hypertrophy, chondrocytes terminally mature and the calcified cartilage matrix is degraded by proteases and is infiltrated by blood vessels. Terminally mature chondrocytes then undergo apoptosis, thus facilitating the remodeling of the vascularized calcified matrix and its invasion by osteoblast precursors. Various families of transcription factors and signaling molecules, including transforming growth factor beta (TGF- $\beta$ ) and Wnts mediate this fine balance of chondrocyte proliferation, hypertrophy and terminal maturation necessary for longitudinal bone growth (Wuelling and Vortkamp, 2010). Further elucidation of the downstream effectors of these pathways may provide insights into the mechanisms controlling the transition of proliferating chondrocytes to hypertrophy.

There is substantial evidence demonstrating that crosstalk between the TGF- $\beta$ and Wnt signals modulate cartilage formation and endochondral ossification (Dong et al., 2005). The repressive activities of TGF- $\beta$ and Wnt signaling on chondrogenesis are mediated by the Twist1 transcription factor. In vitro gain and loss of function studies by Reinhold et al., demonstrated that Twist 1 negatively regulates chondrogenesis and chondrocyte gene expression downstream of activated canonical Wnt signaling (Reinhold et al., 2006). Our previously published studies using in vitro models of chondrocyte maturation, indicated that Twist 1

Abbreviations used in this paper: bHLH, basis helix-loop-helix; Col2, type II collagen; TG, transgenic; BV/TV, bone volume/total volume; PZ, proliferating zone; HZ, hypertrophic zone; $\mu \mathrm{CT}$, micro-computed tomography.

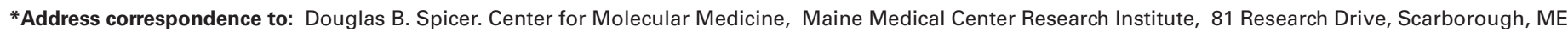
04074, USA. Tel: 1-207- 885-8150. e-mail: spiced@mmc.org

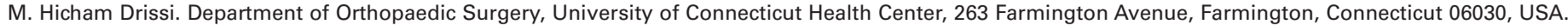
Tel: 1-860-679-6698. Fax: +1-860-679-2109. e-mail: drissi@uchc.edu
}

Supplementary Material (one figure) for this paper is available at: http://dx.doi.org/10.1387/ijdb.103274rg

Accepted: 4 May 2011. Final, author-corrected PDF published online: 7 July 2011

ISSN: Online 1696-3547, Print 0214-6282

(C) 2011 UBC Press

Printed in Spain 
transcripts are down-regulated as chondrocytes mature and that differential regulation of Twist1 and Runx 2 mediate stage-specific cooperative or antagonistic effects of TGF- $\beta$ and canonical Wnt signaling during chondrocyte maturation (Dong et al., 2007). Moreover, Twist1 exerted repressive effects on in vitro chondrocyte maturation (Dong et al., 2007).

TWIST1 haploinsufficiency results in Saethre-Chotzen, a syndrome characterized by craniosynostosis and limb abnormalities (Paznekas et al., 1998). Trisomy of the TWIST1 locus also results in craniofacial defects (Papadopoulou et al., 2006), and interestingly both of these conditions are associated with short stature, indicating that small changes in Twist1 expression have significant effects on skeletal formation and growth. Twist 1 impedes osteoblast differentiation, partially through inhibition of Runx2 activity (Bialek et al., 2004). Moreover, Twist1 plays a role in immature chondrocytes of the perichondrium during skeletal development by regulating Runx2 activation of FGF18 expression (Hinoi et al., 2006). Two studies using several Twist1 mutant alleles demonstrated that incremental reduction of Twist 1 activity during early limb bud patterning alters Shh expression domains and markedly affects limb and girdle development (Krawchuk et al., 2010,, Zhang etal., 2010). Removal of Twist 1 activity from the limb mesenchyme using a floxed conditional null allele ( Twist $^{\text {tx }}$ ) crossed with the Prx-Cre transgene resulted in
A

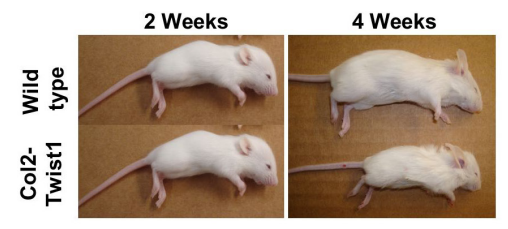

C

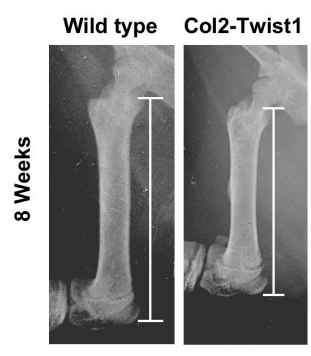

E
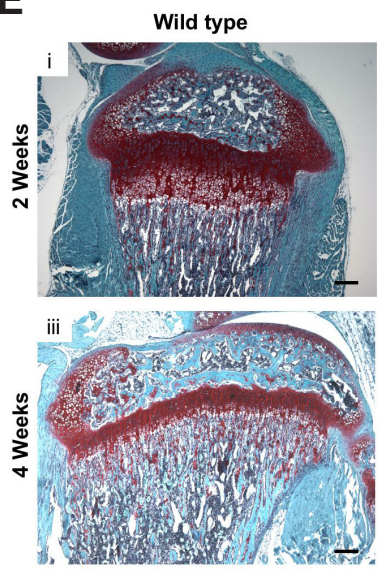

B

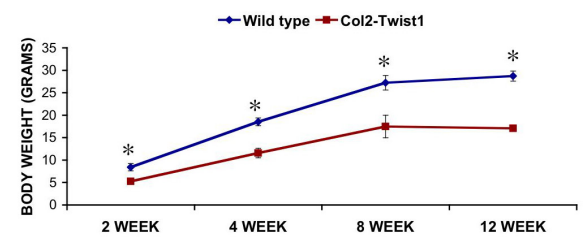

D

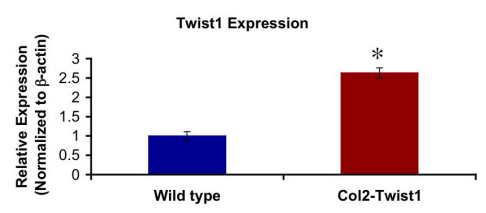

forelimb patterning defects, hypoplastic scapula and clavicle, tibial aplasia and preaxial polydactyly exhibiting disorganized cartilage elements (Krawchuk et al., 2010). These observations raise the possibility of spatial and temporal regulation of cartilage formation by Twist 1 in a dose-dependent manner.

In this study, we assessed the effects of persistent Twist1 expression in chondrocytes in vivo via tissue-specific activation of a Twist 1 transgene in type II collagen-expressing cells. We found that maintenance of Twist 1 expression in mature chondrocytes resulted in postnatal growth retardation and growth plate defects. Moreover, micro-computed tomography $(\mu \mathrm{CT})$ of hindlimbs from Twist 1 transgenic mice further substantiated a role for Twist 1 during endochondral ossification.

\section{Results}

\section{Impaired skeletal growth in mice with persistent chondrocyte- specific Twist 1 expression}

Restricted postnatal expression of Twist1 proteins in immature, proliferating growth plate chondrocytes and its exclusion from hypertrophic chondrocytes (Supplemental Fig. 1), suggested a role for Twist1 in the regulation of skeletal growth and maintenance of cartilage tissue. Moreover, these observations indicated that decreased expression of Twist1 may be required for proper chondrocyte maturation. To test this, we used a Twist 1 transgene that is conditionally activated by Cre recombinase (Chakraborty et al., 2010, Connerney et al., 2008, Connerney et al., 2006). Twist1 mice were crossed with Col2a1-Cre mice, which express Cre in proliferating chondrocytes, to activate constitutive Twist1 expression in these cells and their progeny. Col2-Twist1 mice displayed a runted phenotype as compared to their wild type littermates as early as two weeks after birth (Fig. 1A). Growth curve analysis revealed a significant reduction $(36-41 \%$ reduction versus wild type) in the body mass of Col2-Twist1 transgenics at two weeks $(8.42 \pm 0.80 \mathrm{gm}$, wild type versus $5.25 \pm 0.62 \mathrm{gm}$, transgenic), four weeks $(18.5 \pm 0.87 \mathrm{gm}$, wild type versus $11.6 \pm 1.06$ gm, transgenic) eight weeks (27.2 $\pm 1.62 \mathrm{gm}$, wild type versus $17.5 \pm 2.51 \mathrm{gm}$, transgenic) and twelve weeks $(28.7 \pm 1.11 \mathrm{gm}$, wild type versus $17.1 \pm 0.09 \mathrm{gm}$, transgenic) (Fig.1B). Shortening of endochondral bones in Col2-Twist1 transgenic

Fig. 1. Persistent, chondrocyte-specific Twist1 expression causes growth retardation. (A) Appearance of wild type and runted Col2-Twist1 littermates at two and four weeks. (B) Growth curves of wild type (blue) and Col2-Twist1 mice (red). Mean \pm standard error (SE) from 6-7 mice. ${ }^{*} \mathrm{P}<0.05$ (C) Radiographs of femurs from wild type and Col2-Twist1 mice at eight weeks. (D) Q-PCR analysis of Twist1 mRNA expression in growth plates from two-week wild type (blue) and Col2-Twist1 (red) mice showed increased Twist1 transcript expression in transgenic mice. ${ }^{*} \mathrm{P}<3.6 \times 10^{-5}$. (E). Safranin $O-$ fast green staining of tibia sections from two and four week old mice showed reduced secondary ossification site formation in Col2-Twist1 mice. Scale bar, $200 \mu \mathrm{m}$. 
A
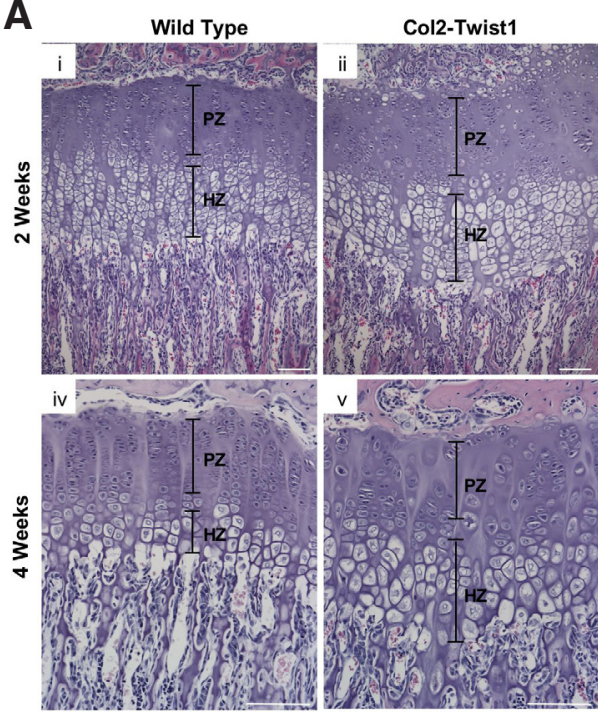

B

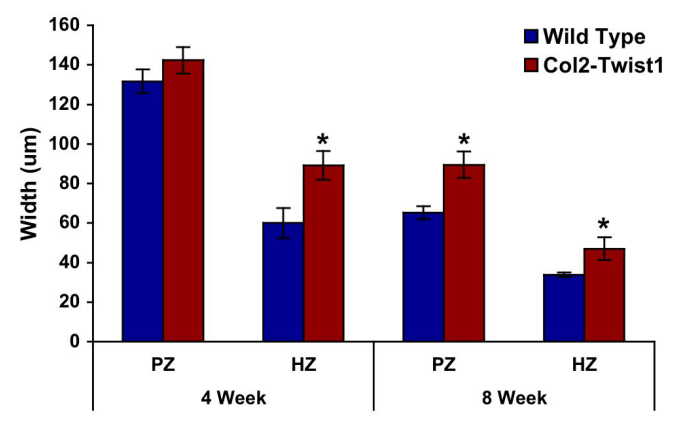

Fig. 2. Aberrant growth plate organization in Col2-Twist1 transgenic mice. (A) H\&E staining revealed hypertrophic zone expansion in the proximal tibia growth plate of Col2-Twist1 mice (ii, iii, v, vi) compared to wild type mice (i, iv), at two (i-iii) and four (iv-vi) weeks. Scale bar, $100 \mu \mathrm{m}$. (B) Widths of proliferating zone (PZ) and hypertrophic zone (HZ) within growth plates of wild type and Col2-Twist1 mice at four and eight weeks determined by histomorphometry. Significantly wider $\mathrm{HZ}$ was observed in transgenic (red) versus wild type mice (blue). ${ }^{*} \mathrm{P}<0.05$.

mice was further confirmed by X-ray analysis (Fig. 1C). At eight weeks, the length of both the femur and tibia were $14.2 \pm 2.9 \%$ ( $p$ $<0.01)$ and $13.5 \pm 2.6 \%(p<0.01)$, respectively shorter in Col2Twist1 transgenics relative to wild type mice (data not shown). Safranin-O/Fast Green staining of proximal tibia sections from two and four week old mice consistently showed that the proportion of cartilage to bone appeared higher in the secondary ossification site of Col2-Twist1 transgenics compared to wild type littermates at two weeks. These differences were not obvious by histology at four weeks, suggesting that secondary ossification site formation was delayed in Col2-Twist1 mice (Fig. 1E).

To evaluate the comparative level of Twist1 expression following transgene activation, we isolated RNA from tissue encompassing the growth plates within the distal femur and proximal tibia of twoweek old Col2-Twist1 transgenic mice and wild type littermates. Q-PCR analysis revealed a $2.63( \pm 0.132)$ fold increase in Twist1 transcript expression in transgenic mice relative to wild type mice $\left(\mathrm{p}<3.6 \times 10^{-5}\right)($ Fig. 1D). Analysis of the proximal tibia of 4-day old
A

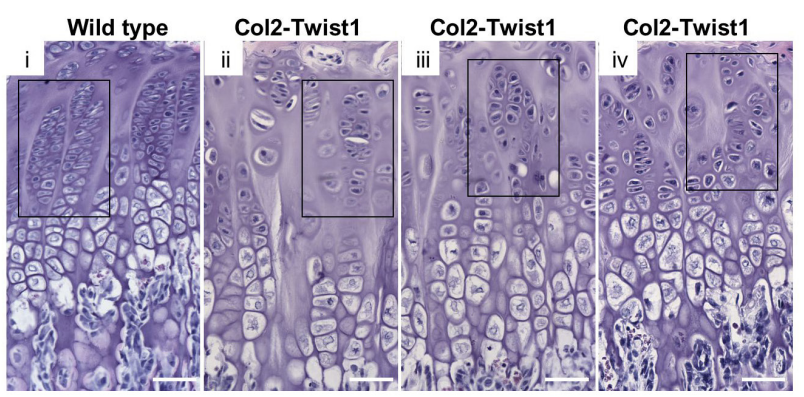

B
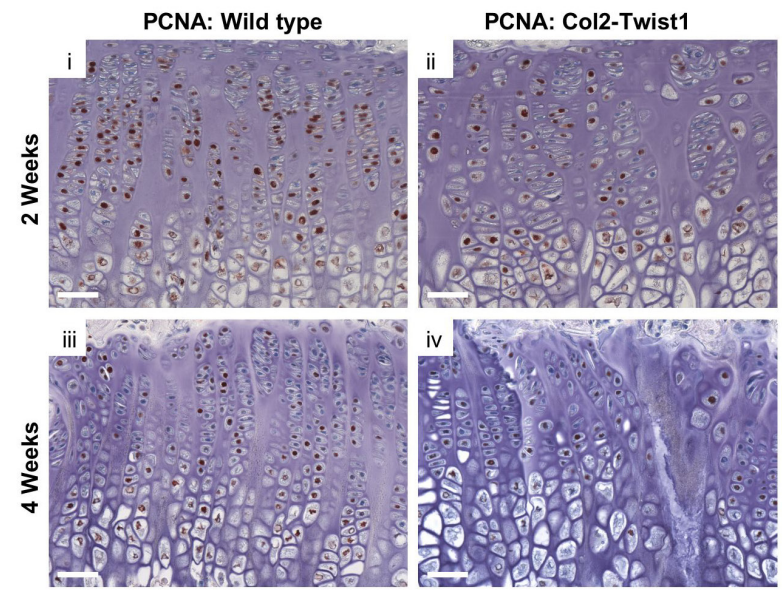

C
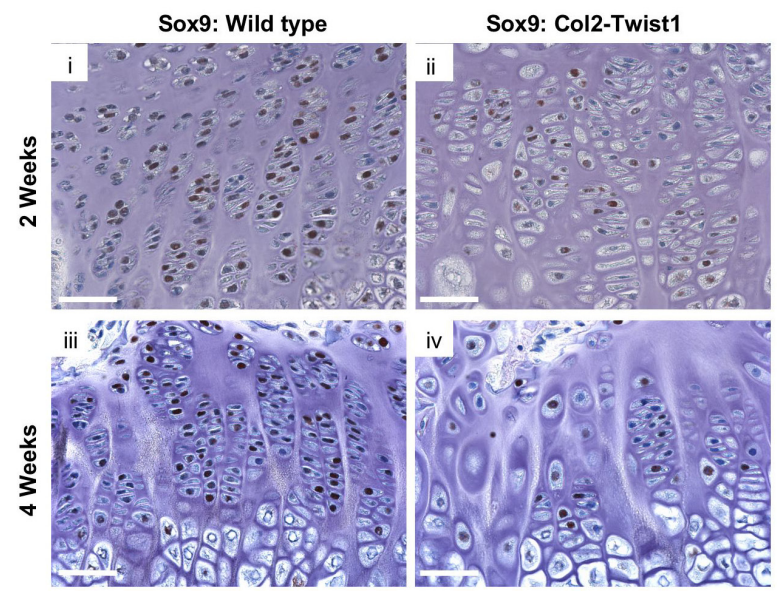

Fig. 3. Growth plate abnormalities are associated with persistent Twist1 expression in chondrocytes. (A) H\&E staining of proximal tibia growth plates from four week old wild type and Col2-Twist1 mice revealed disorderly columnar arrays of proliferating chondrocytes and acellular regions in Col2-Twist1 growth plates. Scale bar, 50 um. (B) Reduced numbers of PCNA positive nuclei in tibia sections of two and four week old Col2-Twist1 mice (ii, iv) as compared to control mice (i, iii). Scale bar, $50 \mathrm{um}$. (C) Immunohistochemical staining showed reduced numbers of Sox9-positive cells in tibia sections from Col2-Twist1 (ii, iv) versus wild type mice (i, iii) at two and four weeks. Scale bar, 50 um.

Col2-Twist1 mice that also contained the R26R reporter, which expresses $\beta$-galactosidase following Cre recombination, indicated transgene activation in all chondrocytes within the growth plate and perichondrium (data not shown). 


\section{Impaired growth plate organization in the Col2-Twist1 trans- genic mice}

We next evaluated the effects of chondrocyte-specific persistent Twist1 expression on growth plate organization and structure. Histological analyses showed that the width of the hypertrophic zone within the proximal tibia growth plate was enlarged in Col2Twist1 transgenic mice compared to wild type littermates at two
A

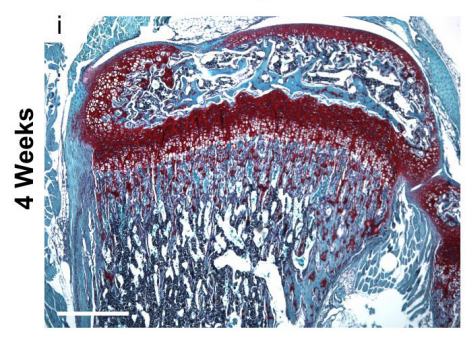

Col2-Twist1

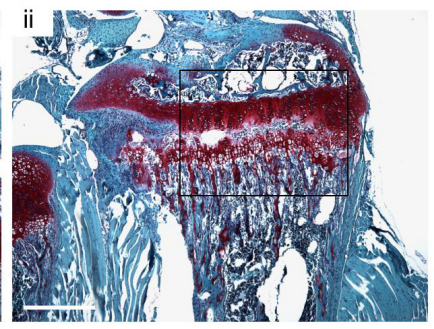

and four weeks (Fig. 2A). These observations were validated by quantitative histomorphometric analyses, revealing significant increases in the width of the tibia growth plate hypertrophic zone in transgenic mice at four weeks $(60.0 \pm 7.59 \mu \mathrm{m}$, wild type mice versus $89.2 \pm 7.25 \mu \mathrm{m}$, Col2-Twist1, $P=0.04)$ and eight weeks $(33.7 \pm 1.20 \mu \mathrm{m}$, wild type versus $47.0 \pm 5.81 \mu \mathrm{m}$, Col2-Twist $1, P$ $=0.05)$ (Fig. 2B). Elevated chondrocyte-specific Twist1 expression

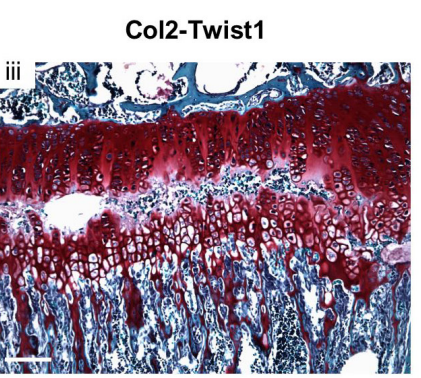
within cartilage also appeared to have a significant effect on the width of the proliferating zone in older (eight weeks) animals (65.3 $\pm 3.27 \mu \mathrm{m}$, wild type versus 89.5 $\pm 6.71 \mu \mathrm{m}$, Col2-Twist1 mice, $P$ $=0.02$ ).

Hematoxylin-eosin (H\&E) staining of the proximal tibia from four-week old wild type mice revealed a growth plate with orderly columns of proliferating chondrocytes (Fig. $3 \mathrm{~A}$ ). In contrast, transgenic tibial growth plates exhibited disorderly columnar arrays of chondrocytes as well as acellular regions in the proliferating zone (Fig. 3A). To address whether the acellularity observed within the growth plates of Col2-Twist1 transgenic mice was attributed to reduced chondrocyte proliferation, immunohistochemical staining for proliferating cell nuclear antigen (PCNA) was performed. These studies showed fewer PCNApositive populations of growth plate chondrocytes (Fig. 3B) in Col2-Twist1 transgenic mice as compared to control mice at two and four weeks. Sox9, which is expressed in all chondroprogenitor cells and differentiated chondrocytes, has also been shown to be critical for the alignment of columnar arrays of proliferating chondrocytes parallel to longitudinal axis of the bone (Akiyama et al., 2002, Bi et al., 1999). Growth plates from the proximal tibias of Col2-Twist1 transgenic mice showed fewer Sox9 immune positive cells versus age-matched wild-type mice (Fig. 3C).

\section{Structural cartilage anomalies in Col2-Twist1 transgenic mice}

Disruption of the typical contiguous columns of growth plate chondrocytes by areas of vascular invasion (Fig. 4A,B) was 
observed within the proliferating zone of the Col2-Twist1 transgenic growth plate, as evidenced by the presence erythrocytes and PECAM (CD31) immune positive cells (Fig. 4B). Immunohistochemical staining for type II collagen expression revealed further evidence of structural anomalies indicative of a vascular response within the transgenic cartilaginous growth plate (Fig. 4C). The growth plates within the proximal tibias of Col2-Twist1 transgenic mice exhibited focal regions of bony deposition emanating from the secondary ossification site (Fig. 4D). Deposition of bony matrix within the proximal tibia growth plate of transgenic mice was confirmed by type I collagen immunostaining (Fig. 4D).

\section{Chondrocyte-specific Twist1 overexpression leads to decrea- sed trabecular bone formation}

In view of the hindlimb shortening and abnormal growth plate organization in Col2-Twist1 transgenic mice, we speculated that elevated chondrocyte-specific Twist1 expression impedes postnatal trabecular bone formation. $\mu \mathrm{CT}$ scans of femurs isolated from wild type and Col2-Twist1 transgenic mice revealed a diminution in trabecular bone formation in Col2-Twist1 transgenic mice at four (Fig. 5A) and eight weeks (Fig. 5B). At four weeks, quantitative $\mu C T$ analysis of femurs showed a significant decrease in bone volume fraction (bone volume/total volume, BV/TV; \%) in Col2Twist 1 transgenics $(2.4 \pm 1.2 \%)$ as compared to control mice (9.4 $\pm 1.8 \%, P=0.007$ ). Similarly, a significant decrease in BV/TV was also observed in transgenic mice $(7.5 \pm 2.4 \%)$ as compared to wild type mice $(11.6 \pm 2.4 \%)$ at eight weeks $(P=0.007)$ (Fig. $5 \mathrm{C}) . \mu \mathrm{CT}$ analysis also showed significant reductions in trabecular thickness in Col2-Twist1 transgenics at four weeks (37 $\pm 2 \mu \mathrm{m}$, wild type versus $29 \pm 2 \mu \mathrm{m}$, transgenic $P=0.02)$ and eight weeks $(41.3 \pm$ $4.2 \mu \mathrm{m}$, wild type mice versus $36 \pm 2.5 \mu \mathrm{m}$ transgenic mice, $P=$ 0.009 ) (Fig. 5D). Trabecular number $(1 / \mathrm{mm})$ was also reduced in Col2-Twist1 transgenic mice at four weeks $(5.11 \pm 0.25 \mu \mathrm{m}$, wild type versus $3.04 \pm 0.64 \mu \mathrm{m}$, transgenic, $P=0.02)$ and eight weeks $(5.12 \pm 0.14 \mu \mathrm{m}$, wild type versus $4 \pm 0.51 \mu \mathrm{m}$, transgenic, $P=$ $0.005)$ (Fig. 5F). Moreover trabecular spacing ( $\mu \mathrm{m})$ was significantly increased in Col2-Twist 1 transgenic mice relative to wild type mice at eight weeks $(194 \pm 6.69 \mu \mathrm{m}$, wild type versus $236 \pm 26.58 \mu \mathrm{m}$, transgenic, $P=0.005$ ) (Fig. $5 \mathrm{E}$ ), further validating the reduction in trabecular bone formation in transgenic mice. These morphometric measurements demonstrated that mice with persistent Twist1 expression in chondrocytes develop a runted phenotype as a result of impaired endochondral ossification.

\section{Discussion}

Gene expression data and studies using normal and diseased cartilage suggest that Twist1 is a key regulator of chondrocyte function (Dong et al., 2007, Karlsson et al., 2010). Genetic studies have sought to define the possible function of Twist 1 during cartilage and limb development. Hinoi et al., previously reported high levels of Twist 1 expression within immature perichondrial cells in vivo, but failed to detect Twist1 transcripts in mature chondrocytes by in situ hybridization (Hinoi et al., 2006). Moreover, their histological analyses of the developing ribs and limbs in $\alpha 1$ (II) Collagen-Cre; Twist $1^{\text {fx }}$ embryos (E13.5 and E16.5), showed no obvious phenotype attributable to Twist1 gene inactivation in chondrocytes (Hinoi et al., 2006). However, conditional Twist1
A

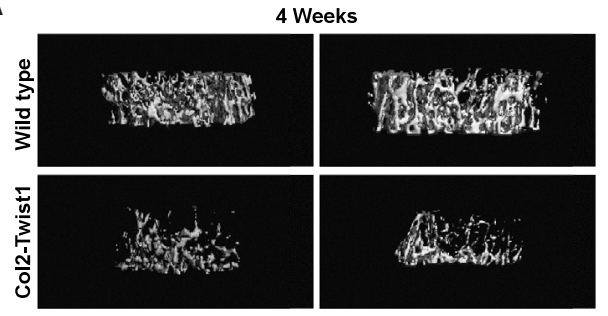

B

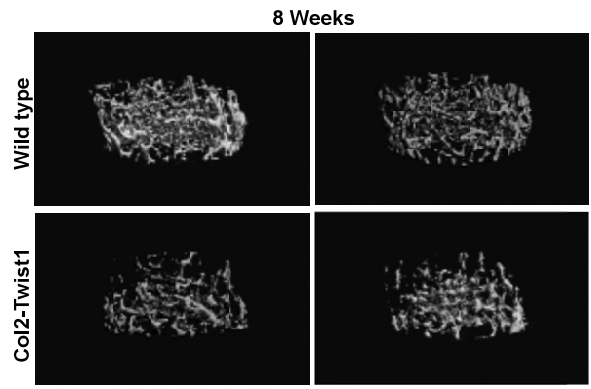

C
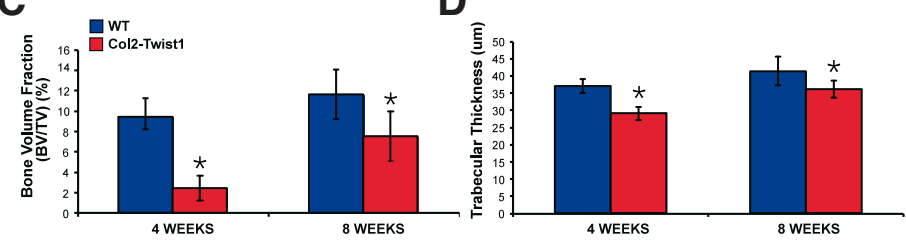

$\mathbf{F}$

E
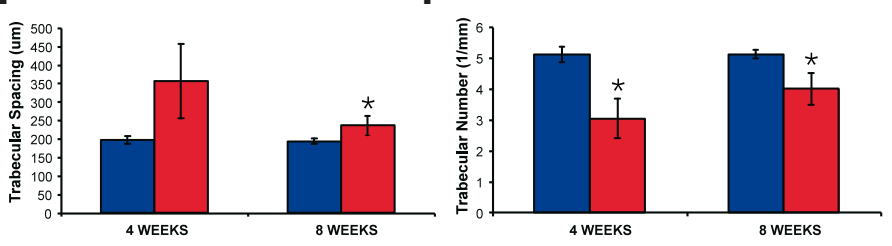

G

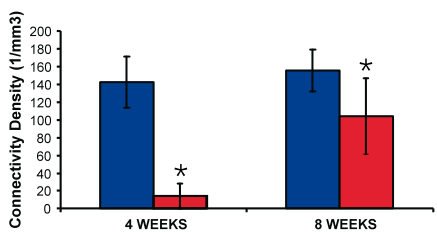

Fig. 5. Persistent chondrocyte-specific Twist1 expression causes postnatal decrease in trabecular bone formation. $\mu C T$ scans of wild type and transgenic femurs at four (A) and eight weeks (B) illustrate reduced trabecular bone volume and density in Col2-Twist1 mice. (C-G) Quantitative analysis reveals significant reduction in bone volume fraction (C); trabecular thickness (D); trabecular number (F); and connectivity density (G) in Col2Twist1 mice (red) versus wild type mice (b/ue) at four and eight weeks. Col2-Twist1 mice displayed increased trabecular spacing (E) versus wild type mice at four and eight weeks ${ }^{*} \mathrm{P}<0.05$. 
abrogation from the limb mesenchyme by crossing Twist $1^{\text {fx }}$ mice with mice containing the Prx1-Cre transgene, which activates Cre expression in prechondrocytes, resulted in forelimb patterning defects, hypoplastic scapula and clavicle, tibial aplasia, and preaxial polydactyly characterized by severe disorganization of cartilage elements (Krawchuk et al., 2010, Zhang et al., 2010). We speculated that the temporal requirement for Twist1 during postnatal chondrocyte formation may provide novel insight into stage- and dose-dependent roles of Twist1 during cartilage maturation and maintenance. Thus, we hypothesized that sustained expression of Twist 1 in chondrocytes during embryonic and postnatal development will delay chondrocyte differentiation and impair endochondral ossification.

The rationale for examining the effects of persistent Twist1 expression on cartilage development in vivo stems from our in vitro functional studies demonstrating that Twist1 acts as a potent inhibitor of chondrocyte hypertrophy (Dong et al., 2007). Others have shown that ectopic expression of Twist 1 in the murine prechondrogenic ATDC5 cell line repressed chondrocyte gene expression and activity; whereas Twist1 knockdown by RNA interference increased the expression of early chondrogenic gene markers (Reinhold et al., 2006). Moreover, using human primary mesenchymal stem cells (MSC), Isenmann et al., demonstrated that enforced expression of Twist1 sustained an immature stromal phenotype and inhibited the chondrogenic potential of human MSCs, as shown by a reduced capacity to synthesize glycosaminoglycans and down-regulated expression of Sox9and collagen type $X$ (Isenmann et al., 2009). Increased expression of different forms of Twist1 in mesenchymal progenitors of the limb resulted in runted limb growth (Firulli et al., 2007), however the phenotype of the limb cartilage of these mice was not analyzed. Thus, we initiated our studies to explore the dose-dependant and temporal function of Twist1 during cartilage formation and maturation in vivo. We used a conditional Cre-induced transgene approach to constitutively express Twist 1 in collagen II-expressing cells and their progeny. Activation of the transgene resulted in a modest ( 2.6 fold) increase in Twist 1 transcripts over endogenous Twist 1 expression, which was the same level as found using this transgene in other tissues (Chakraborty et al., 2010), yet the expression of the transgene was maintained as chondrocytes matured. Sustained expression of Twist1 in cartilage led to a growth phenotype, characterized by shortening of the limbs and reduced body mass. Various Twist 1 loss of function studies have also reported growth abnormalities and limb dismorphogenesis (Firulli et al., 2005, Firulli et al., 2007, Krawchuk et al., 2010, Zhang et al., 2010), thus demonstrating that in addition to its established function during intramembranous bone formation, Twist1 also plays a role in endochondral ossification.

Histological assessments of the proximal tibias from Col2-Twist1 transgenic mice showed a disruption of the regular columnar arrangement of the proliferating and hypertrophic chondrocytes. Immunohistochemical analyses further revealed decreased PCNA and Sox9 stained populations of growth plate chondrocytes in Col2-Twist1 transgenic mice. Sox9, expressed by all chondroprogenitor cells and differentiating chondrocytes, is critical for the expression of components of the cartilaginous ECM (Bi et al., 1999) and promotes chondrocyte proliferation and alignment of the proliferative clones into columnar arrays parallel to longitudinal axis of bone (Akiyama et al., 2002). Disruption of the growth plate chondrocyte columnar arrays in Col2-Twist1 transgenic mice may be attributed to reduced Sox9 expression and/or insufficient number of proliferating growth plate chondrocytes.

While an effect of increased Twist1 expression was predicted to inhibit Sox9 expression, and thus indirectly affect chondrocyte proliferation (Akiyama et al., 2002), Col2-Twist1 transgenic mice also displayed an unexpected expansion of the hypertrophic zone in postnatal growth plates. It is plausible that accumulation of hypertrophic chondrocytes in vivo may be attributed to delayed chondrocyte terminal maturation and apoptosis induced by ossification fronts. However, we did not observe decreased apoptosis in Col2-Twist1 mice (data not shown). Chondrocyte hypertrophy and subsequent ossification is coordinately regulated by Runx2 and Mef2c transcription factors (Wuelling and Vortkamp, 2010). Interestingly, both of these factors are direct targets of Twist1 inhibition (Bialek et al., 2004, Spicer et al., 1996), which could explain the phenotype of the Col2-Twist1 mice. Importantly, all of the in vitro studies identifying an important role for Twist1 in chondrocyte specification and early differentiation events were done by expressing Twist1 in MSCs or prechondrocytes, while Col2-Twist1 mice initiate expression of Twist1 at a later stage of chondrocyte differentiation, which may have allowed us to identify a later role for Twist 1 in chondrocyte differentiation. These studies indicate that deregulation of Twist1 expression within the growth plate negatively impacts endochondral ossification.

Histologic and immunohistochemical analyses revealed additional growth plate abnormalities in Col2-Twist1 transgenics, including blood vessel and focal bony formation within the cartilaginous matrix. Postnatal chondrocyte-specific ablation of Ihh (Maeda et al., 2007) led to similar features as Col2-Twist1 mice, including growth retardation, decreased PCNA-stained chondrocytes, loss of chondrocyte columnar organization and aberrant vascular invasion within the growth plate, attributed to the premature differentiation of mutant chondrocytes. However, $\mu \mathrm{CT}$ analyses of femurs from wild type and Col2-Twist1 transgenic mice showed that chondrocyte-specific Twist1 expression led to significant decreases in bone volume fraction, trabecular thickness, trabecular number and connectivity density. These findings argue that aberrant vascular invasion and bony bridge trabeculae within Col2-Twist1 transgenic growth plates are not due to advanced replacement of mineralized cartilage by bone. Rather, we postulate that these defects arise due to growth plate fracture or trauma. In support of this, animal models of growth plate fracture have displayed structural disorganization, formation of vertical septa, bone bridge formation and longitudinal shortening (Wattenbarger et al., 2002). Moreover, marrow-derived osteogenic precursors are recruited to undergo intermembranous bone formation at the growth plate defect site (Xian et al., 2004). We speculate that Col2-Twist1 transgenic growth plate may be prone to trauma, due to impaired cartilage ECM integrity or production, resulting in decreased trabecular bone formation.

As previous studies have not addressed Twist1 function in postnatal chondrocyte development, the effects of chondrocytespecific loss of Twist1 function in postnatal development warrant further investigation. While future experiments will explore the cellular and molecular mechanisms underlying Twist 1 function during chondrocyte differentiation, our findings argue that regulated expression of Twist1 within growth plate chondrocytes is important for proper endochondral ossification. 


\section{Materials and Methods}

\section{Mice and genotyping}

Female CAGCAT-Twist1 mice were bred with Col2a1-Creor with Col2a1Cre;R26R males to generate offspring heterozygous for each transgene. Col2a1-Cre and R26R mice were obtained from The Jackson Laboratory, Bar Harbor, ME. Genotyping for the Cre and Twist1 transgenes was performed by PCR of genomic DNA from 3-week postnatal tail clips using Cre, $C A T$ and $L a c Z$ specific primers (Connerney et al., 2008).

\section{RNA isolation and real-time PCR}

Growth plates from Col2-Twist1 and wild type mice were homogenized in TRIzol reagent (Invitrogen) using the TissueLyserll (Qiagen). Total RNAwas extracted, reverse transcribed and Q-PCR performed using mouse specific primers for Twist1 (forward primer, 5'-GCAGTGGTGGAATGCCTTTA-3'; reverse primer, 5'-TGTGGTATGGCTGATTATGATCTC-3'), and 9-actin (forward primer, 5'-AGATGTGGATCAGCAAGCAG-3'; reverse primer, 5'-GCGCAAGTTAGGTTTTGTCA-3'). Data was analysis was performed using the StepOne Software v2.1.

\section{Histological staining}

Hindlimbs from 2, 4 and 8 week old mice were dissected and fixed in $4 \%$ paraformaldehyde for $2-5$ days at $4^{\circ} \mathrm{C}$. Specimens were decalcified in $15 \%$ EDTA ( $\mathrm{pH} 7.1$ ) for 5 days. Samples were embedded in paraffin and 5um sections were collected. Sections were deparaffinized in xylene and rehydrated through graded alcohols to water, then stained with hemotoxylin-eosin, Safranin O/Fast green or $1 \%$ Alcian blue $(\mathrm{pH} 2.5)$ (PolyScientific).

\section{Immunohistochemical studies}

Deparaffinized sections were first incubated in antigen retrieval solution (4N HCL or pepsin) for 15 minutes at $37^{\circ} \mathrm{C}$. Endogenous peroxidase activity was quenched with $3 \%$ hydrogen peroxide for 30 minutes. Sections were permeabilized in $0.1 \%$ Triton-X-100 and non-specific binding sites were blocked with $1 \%$ bovine serum albumin and $10 \%$ goat serum in PBS. Sections were incubated with or without primary antibodies, including mouse anti-collagen type II (1:200, Millipore), rabbit anti-PCNA (1:200, Abcam), rabbit anti-Sox9 (1:75, Abcam), mouse anti-Twist1 3E11 (1:50, Novus Biologicals) and goat anti-PECAM (1:75, Santa Cruz). Labeling was visualized with the appropriate peroxidase-conjugated secondary antibody and Zymed Aminoethyl Carbazole Substrate Kit (Invitrogen). Image acquisition used a Q-Imaging Retig 2000R camera connected to Nikon Eclipse 50i microscope and image analyses performed using NIS Elements B\$ 3.0 software.

\section{$X$-ray and micro-computed tomography}

Femurs were disarticulated at the hip and knee, dissected of soft tissue, and fixed in $10 \%$ formalin. Bone morphometry was measured using micro-focus conebeam X-ray computed tomography ( $\mu$ CT40, Scanco Medical AG, Switzerland). Scanning was performed at $55 \mathrm{kV}$ and $145 \mu \mathrm{A}$, collecting 1000 projections/rotation at $300 \mathrm{msec}$ integration time. Threedimensional images were reconstructed using standard convolution and back-projection algorithms with Gausian filtering, and rendered within a $12.3 \mathrm{~mm}$ field of view at 578,704 voxels $/ \mathrm{mm}^{3}$ (isometric $12 \mathrm{~mm}$ voxels). Standard algorithms describing trabecular morphometry were applied for measures of bone architecture and mineral density.

\section{Acknowledgements}

This work was supported by the University of Connecticut Health Center. Additional support was provided by the Maine Medical Center Research Institute Histopathology Core Facility funded by NIH grant P20 RR18789 (Dr. D. Wojchowski).

\section{References}

AKIYAMA, H, CHABOISSIER, MC, MARTIN, JF, SCHEDL, A and DE CROMBRUGGHE, B (2002). The transcription factor Sox 9 has essential roles in successive steps of the chondrocyte differentiation pathway and is required for expression of Sox5 and Sox6. Genes Dev 16: 2813-2828.

BI, W, DENG, JM, ZHANG, Z, BEHRINGER, RR and DE CROMBRUGGHE, B (1999) Sox9 is required for cartilage formation. Nature Genetics 22: 85-89.

BIALEK, P, KERN, B, YANG, X, SCHROCK, M, SOSIC, D, HONG, N, WU, H, YU, K, ORNITZ, DM, OLSON, EN et al., (2004). A twist code determines the onset of osteoblast differentiation. Dev Cell 6: 423-435.

CHAKRABORTY, S, WIRRIG, EE, HINTON, RB, MERRILL, WH, SPICER, DB and YUTZEY, KE (2010). Twist1 promotes heart valve cell proliferation and extracellular matrix gene expression during development in vivo and is expressed in human diseased aortic valves. Dev Biol 347: 167-179.

CONNERNEY, J, ANDREEVA, V, LESHEM, Y, MERCADO, MA, DOWELL, K, YANG, $X$, LINDNER, V, FRIESEL, RE and SPICER, DB (2008). Twist1 homodimers enhance FGF responsiveness of the cranial sutures and promote suture closure. Dev Biol 318: 323-334.

CONNERNEY, J, ANDREEVA, V, LESHEM, Y, MUENTENER, C, MERCADO, MAand SPICER, DB (2006). Twist1 dimer selection regulates cranial suture patterning and fusion. Dev Dyn 235: 1345-1357.

DONG, Y, DRISSI, H, CHEN, M, CHEN, D, ZUSCIK, MJ, SCHWARZ, EM and O'KEEFE, RJ (2005). Wnt-mediated regulation of chondrocyte maturation modulation by TGF-beta. J Cell Biochem 95: 1057-1068.

DONG, YF, SOUNG DY, CHANG, Y, ENOMOTO-IWAMOTO, M, PARIS, M, O'KEEFE, RJ, SCHWARZ, EM and DRISSI, H (2007). Transforming growth factor-beta and Wnt signals regulate chondrocyte differentiation through Twist1 in a stage-specific manner. Mol Endocrinol 21: 2805-2820.

FIRULLI, BA, KRAWCHUK, D, CENTONZE, VE, VARGESSON, N, VIRSHUP, DM CONWAY, SJ, CSERJESI, P, LAUFER, E and FIRULLI, AB (2005). Altered Twist1 and Hand2 dimerization is associated with Saethre-Chotzen syndrome and limb abnormalities. Nat Genet 37: 373-381.

FIRULLI, BA, REDICK, BA, CONWAY, SJ and FIRULLI, AB (2007). Mutations within helix I of Twist1 result in distinct limb defects and variation of DNA binding affinities. J Biol Chem 282: 27536-27546.

HINOI, E, BIALEK, P, CHEN, YT, RACHED, MT, GRONER, Y, BEHRINGER, RR, ORNITZ, DM and KARSENTY, G (2006). Runx2 inhibits chondrocyte proliferation and hypertrophy through its expression in the perichondrium. Genes Dev 20: $2937-2942$.

ISENMANN, S, ARTHUR, A, ZANNETTINO, AC, TURNER, JL, SHI, S, GLACKIN, CA and GRONTHOS, S (2009). TWIST family of basic helix-loop-helix transcription factors mediate human mesenchymal stem cell growth and commitment. Stem Cells 27: 2457-2468.

KARLSSON, C, DEHNE, T, LINDAHL, A, BRITTBERG, M, PRUSS, A, SITTINGER $M$ and RINGE, J (2010). Genome-wide expression profiling reveals new candidate genes associated with osteoarthritis. Osteoarthritis Cartilage 18: 581-592.

KRAWCHUK, D, WEINER, SJ, CHEN, YT, LU, B, COSTANTINI, F, BEHRINGER, RR and LAUFER, E (2010). Twist1 activity thresholds define multiple functions in limb development. Dev Biol 347: 133-146.

MAEDA, Y, NAKAMURA, E, NGUYEN, MT, SUVA, LJ, SWAIN, FL, RAZZAQUE, MS MACKEM, S and LANSKE, B (2007). Indian Hedgehog produced by postnatal chondrocytes is essential for maintaining a growth plate and trabecular bone. Proc Natl Acad Sci USA 104: 6382-6387.

PAPADOPOULOU, E, SIFAKIS, S, SARRI, C, GYFTODIMOU, J, LIEHR, T, MRASEK, $\mathrm{K}, \mathrm{KALMANTI}, \mathrm{M}$ and PETERSEN, MB (2006). A report of pure 7p duplication syndrome and review of the literature. Am J Med Genet 140: 2802-2806.

PAZNEKAS, WA, CUNNINGHAM, ML, HOWARD, TD, KORF, BR, LIPSON, MH, GRIX, AW, FEINGOLD, M, GOLDBERG, R, BOROCHOWITZ, Z, ALECK, K et al., (1998). Genetic heterogeneity of Saethre-Chotzen syndrome, due to TWIST and FGFR mutations. Am J Hum Genet 62: 1370-1380.

REINHOLD, MI, KAPADIA, RM, LIAO, Z and NASKI, MC (2006). The Wnt-inducible transcription factor Twist1 inhibits chondrogenesis. J Biol Chem 281: 1381-1388. 
648 R.M. Guzzo et al.

SPICER, DB, RHEE, J, CHEUNG, WL and LASSAR, AB (1996). Inhibition of myogenic bHLH and MEF2 transcription factors by the bHLH protein Twist. Science 272: $1476-1480$.

WATTENBARGER, JM, GRUBER, HE and PHIEFFER, LS (2002). Physeal fractures, part I: histologic features of bone, cartilage, and bar formation in a small animal model. J Pediatr Orthop 22: 703-709.

WUELLING, M and VORTKAMP, A (2010). Transcriptional networks controlling chondrocyte proliferation and differentiation during endochondral ossification.
Pediatr Nephrol 25: 625-631

XIAN, CJ, ZHOU, FH, MCCARTY, RC and FOSTER, BK (2004). Intramembranous ossification mechanism for bone bridge formation at the growth plate cartilage injury site. J Orthop Res 22: 417-426.

ZHANG, Z, SUI, P, DONG, A, HASSELL, J, CSERJESI, P, CHEN, YT, BEHRINGER, RR and SUN, $X$ (2010). Preaxial polydactyly: interactions among ETV, TWIST1 and HAND2 control anterior-posterior patterning of the limb. Development 137 3417-3426. 